\title{
The Existence of Solutions to a System of Discrete Fractional Boundary Value Problems
}

\author{
Yuanyuan Pan, Zhenlai Han, Shurong Sun, and Yige Zhao
}

School of Mathematical Sciences, University of Jinan, Jinan, Shandong 250022, China

Correspondence should be addressed to Zhenlai Han, hanzhenlai@163.com

Received 7 December 2011; Revised 26 December 2011; Accepted 1 January 2012

Academic Editor: Ferhan M. Atici

Copyright (C) 2012 Yuanyuan Pan et al. This is an open access article distributed under the Creative Commons Attribution License, which permits unrestricted use, distribution, and reproduction in any medium, provided the original work is properly cited.

We study the existence of solutions for the boundary value problem $-\Delta^{v} y_{1}(t)=f\left(y_{1}(t+v-1), y_{2}(t+\right.$ $\mu-1)),-\Delta^{\mu} y_{2}(t)=g\left(y_{1}(t+v-1), y_{2}(t+\mu-1)\right), y_{1}(v-2)=\Delta y_{1}(v+b)=0, y_{2}(\mu-2)=\Delta y_{2}(\mu+b)=$ 0 , where $1<\mu, v \leq 2, f, g: \mathbb{R} \times \mathbb{R} \rightarrow \mathbb{R}$ are continuous functions, $b \in \mathbb{N}_{0}$. The existence of solutions to this problem is established by the Guo-Krasnosel'kii theorem and the Schauder fixedpoint theorem, and some examples are given to illustrate the main results.

\section{Introduction}

In recent years, fractional differential equations have been of great interest. It is caused both by the intensive development of the theory of fractional calculus itself and by the applications of such constructions in various sciences such as physics, mechanics, chemistry, and engineering. The continuous fractional calculus has seen tremendous growth within the last ten years or so. Some of the recent progress in the continuous fractional calculus has included a paper [1] in which the authors explored a continuous fractional boundary value problem of conjugate type. Using cone theory, they then deduced the existence of one or more positive solutions. Other recent work in the direction of those articles may be found, see $[2-14]$.

Recently, there appeared a number of papers on the discrete fractional calculus, such as [15-32], which has helped to build up some of the basic theories of this area. For example, Atici and Eloe discussed properties of the generalized falling function, a corresponding power rule for fractional delta-operators, and the commutivity of fractional sums in [15]. And Goodrich studied a two-point fractional boundary value problem in [24], which gave the existence results for a certain two-point boundary value problem of right-focal type for a fractional difference equation. 
From the above works, we can see a fact, although the discrete fractional boundary value problem has been studied by some authors, to the best of our knowledge, systems of discrete fractional boundary value problem are seldom considered.

Goodrich in [24] considered a discrete fractional boundary value problem

$$
\begin{aligned}
& -\Delta^{v} y(t)=f(\mathrm{t}+v-1, y(t+v-1)), \\
& y(v-2)=0=\Delta y(v+b),
\end{aligned}
$$

where $t \in[0, b+1]_{\mathbb{N}_{0}}, f:[v-1, v+b-1]_{\mathbb{N}_{v-1}} \times \mathbb{R} \rightarrow \mathbb{R}$, and $1<v \leq 2$, which gave a representation for the solution to this problem and established the existence and uniqueness of solution to this problem by the Guo-Krasnosel'kii theorem.

Goodrich in [25] examined a system of discrete fractional difference equations subject to nonlocal boundary conditions

$$
\begin{array}{ll}
-\Delta^{v_{1}} y_{1}(t)=\lambda_{1} a_{1}\left(t+v_{1}-1\right) f_{1}\left(y_{1}\left(t+v_{1}-1\right),\right. & \left.y_{2}\left(t+v_{2}-1\right)\right), \\
-\Delta^{v_{2}} y_{2}(t)=\lambda_{2} a_{2}\left(t+v_{2}-1\right) f_{2}\left(y_{1}\left(t+v_{1}-1\right),\right. & \left.y_{2}\left(t+v_{2}-1\right)\right),
\end{array}
$$

for $t \in[0, b]_{\mathbb{N}_{0}}$, subject to the conditions

$$
\begin{array}{ll}
y_{1}\left(v_{1}-2\right)=\psi_{1}\left(y_{1}\right), & y_{2}\left(v_{2}-2\right)=\psi_{2}\left(y_{2}\right), \\
y_{1}\left(v_{1}+b\right)=\phi_{1}\left(y_{1}\right), & y_{2}\left(v_{2}+b\right)=\phi_{2}\left(y_{2}\right),
\end{array}
$$

where $\lambda_{i}>0, a_{i}: \mathbb{R} \rightarrow[0,+\infty), v_{i} \in(1,2]$, and for each $i=1,2, \psi_{i}, \phi_{i}: \mathbb{R}^{b+3} \rightarrow \mathbb{R}$ are given functionals, and $f_{i}:[0,+\infty) \times[0,+\infty) \rightarrow[0,+\infty)$ are continuous for each admissible $i$. This paper gives the existence of a positive solution on discrete fractional boundary value problems.

Motivated by all the works above, in this paper, we discuss the existence of solutions to a system of discrete fractional boundary value problem

$$
\begin{array}{lll}
-\Delta^{v} y_{1}(t)=f\left(y_{1}(t+v-1),\right. & \left.y_{2}(t+\mu-1)\right), & \text { for } t \in[0, b+1]_{\mathbb{N}_{0}} \\
-\Delta^{\mu} y_{2}(t)=g\left(y_{1}(t+v-1),\right. & \left.y_{2}(t+\mu-1)\right), & \text { for } t \in[0, b+1]_{\mathbb{N}_{0}} \\
y_{1}(v-2) & =\Delta y_{1}(v+b)=0, & \\
y_{2}(\mu-2)=\Delta y_{2}(\mu+b)=0, &
\end{array}
$$

where $1<\mu, v \leq 2, f, g: \mathbb{R} \times \mathbb{R} \rightarrow \mathbb{R}$ are continuous functions, $b \in \mathbb{N}_{0}$.

The plan of the paper is as follows. In Section 2, we will present some lemmas in order to prove our main results. Section 3, establishes and proves our main results. In Section 4 we give some examples to illustrate the main results.

\section{Preliminaries}

For the convenience of the reader, we give some background materials from fractional difference theory to facilitate the analysis of problem (1.4). These and other related results and their proof can be found in [15-18]. 
Definition 2.1. One defines $t^{\underline{v}}:=\Gamma(t+1) / \Gamma(t+1-v)$, for any $t$ and $v$ for which the right-hand side is defined. One also appeals to the convention that if $t+1-v$ is a pole of the Gamma function and $t+1$ is not a pole, then $t^{\underline{v}}=0$.

Definition 2.2. The $v$ th fractional sum of a function $f$, for $v>0$, is defined by

$$
\Delta^{-v} f(t ; a):=\frac{1}{\Gamma(v)} \sum_{s=a}^{t-v}(t-s-1)^{\frac{v-1}{v}} f(s)
$$

for $t \in\{a+v, a+v+1, \ldots\}:=\mathbb{N}_{a+v}$. One also defines the $v$ th fractional difference for $v>0$ by $\Delta^{v} f(t):=\Delta^{N} \Delta^{v-N} f(t)$, where $t \in \mathbb{N}_{a+v}$ and $v \in \mathbb{N}$ is chosen so that $0 \leq N-1<v \leq N$.

Lemma 2.3. Let $t$ and $v$ be any numbers for which $t^{\underline{v}}$ and $t^{\underline{v-1}}$ are defined, then

$$
\Delta t^{\underline{v}}=v t^{\underline{v-1}}
$$

Lemma 2.4. Let $0 \leq N-1<v \leq N$, where $N \in \mathbb{N}$ and $N-1 \geq 0$, then

$$
\Delta^{-v} \Delta^{v} y(t)=y(t)+C_{1} t^{\frac{v-1}{2}}+C_{2} t^{\frac{v-2}{2}}+\cdots+C_{N} t^{\nu-N},
$$

for some $C_{i} \in \mathbb{R}$, with $1 \leq i \leq N$.

Before stating the next useful lemma, let us introduce the following notation, which will be important in the sequel:

$$
\begin{aligned}
T_{1} & :=\left\{(t, s) \in[v-1, v+b+1]_{\mathbb{N}_{v-1}} \times[0, b+1]_{\mathbb{N}_{0}}: 0 \leq s<t-v+1 \leq b+2\right\}, \\
T_{2} & :=\left\{(t, s) \in[v-1, v+b+1]_{\mathbb{N}_{v-1}} \times[0, b+1]_{\mathbb{N}_{0}}: 0 \leq t-v+1 \leq s \leq b+2\right\}, \\
L_{1} & :=\left\{(t, s) \in[\mu-1, \mu+b+1]_{\mathbb{N}_{\mu-1}} \times[0, b+1]_{\mathbb{N}_{0}}: 0 \leq s<t-\mu+1 \leq b+2\right\}, \\
L_{2} & :=\left\{(t, s) \in[\mu-1, \mu+b+1]_{\mathbb{N}_{\mu-1}} \times[0, b+1]_{\mathbb{N}_{0}}: 0 \leq t-\mu+1 \leq s \leq b+2\right\} .
\end{aligned}
$$

Lemma 2.5. ([24]) The unique solution of the discrete fractional boundary value problem

$$
-\Delta^{v} y(t)=h(t+v-1), \quad y(v-2)=0=\Delta y(v+b)
$$

is given by

$$
y(t):=\sum_{s=0}^{b+1} G_{1}(t, s) h(s+v-1)
$$


where $1<v \leq 2, G_{1}(t, s)$ is given by

$$
G_{1}(t, s):=\frac{1}{\Gamma(v)} \begin{cases}\frac{\Gamma(b+3) t^{\frac{v-1}{}}}{\Gamma(v+b+1)}(v+b-s-1)^{\frac{v-2}{2}-(t-s-1)^{\frac{v-1}{2}},} & (t, s) \in T_{1}, \\ \frac{\Gamma(b+3) t^{\frac{v-1}{}}}{\Gamma(v+b+1)}(v+b-s-1)^{\frac{v-2}{2}}, & (t, s) \in T_{2} .\end{cases}
$$

Remark 2.6. Let us note that in case we put $\mu$ replacing $v$ in Lemma 2.5 , it follows that

$$
y(t):=\sum_{s=0}^{b+1} G_{2}(t, s) h(s+\mu-1),
$$

where $1<\mu \leq 2, G_{2}(t, s)$ is given by

$$
G_{2}(t, s):=\frac{1}{\Gamma(\mu)} \begin{cases}\frac{\Gamma(b+3) t \frac{\mu-1}{\Gamma(\mu+b+1)}}{\Gamma(\mu+b-s-1) \frac{\mu-2}{-}-(t-s-1) \frac{\mu-1}{},} & (t, s) \in L_{1}, \\ \frac{\Gamma(b+3) t \frac{\mu-1}{\Gamma(\mu+b+1)}}{\Gamma+}(\mu+b-s-1) \frac{\mu-2}{}, & (t, s) \in L_{2} .\end{cases}
$$

Lemma 2.7. ([24]) The Green function $G_{1}(t, s)$ given in Lemma 2.5 satisfies

(i) $G_{1}(t, s) \geq 0$ for $(t, s) \in[v-1, v+b+1]_{\mathbb{N}_{v-1}} \times[0, b+1]_{\mathbb{N}_{0}}$

(ii) $\max _{t \in[v-1, v+b+1]_{\mathbb{v}-1}} G_{1}(t, s)=G_{1}(s+v-1, s)$, for $s \in[0, b]_{\mathbb{N}_{0}}$

(iii) there exists a number $\gamma_{1} \in(0,1)$ such that

$\min _{t \in[(b+v) / 4,3(b+v) / 4]} G_{1}(t, s) \geq \gamma_{1} \max _{t \in[v-1, v+b+1]_{\mathbb{N}-1}} G_{1}(t, s)=\gamma_{1} G_{1}(s+v-1, s)$, for $s \in[0, b+1]_{\mathbb{N}_{0}}$.

Similarly, $G_{2}(t, s)$ satisfies

(I) $G_{2}(t, s) \geq 0$ for $(t, s) \in[\mu-1, \mu+b+1]_{\mathbb{N}_{\mu-1}} \times[0, b+1]_{\mathbb{N}_{0}}$

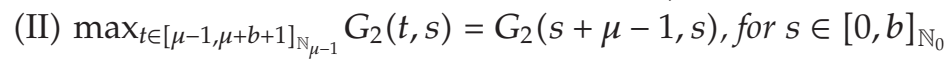

(III) there exists a number $\gamma_{2} \in(0,1)$ such that

$\min _{t \in[(b+\mu) / 4,3(b+\mu) / 4]} G_{2}(t, s) \geq \gamma_{2} \max _{t \in[\mu-1, \mu+b+1]_{\mathbb{N}_{\mu-1}}} G_{2}(t, s)=\gamma_{2} G_{2}(s+\mu-1, s)$, for $s \in[0, b+1]_{\mathbb{N}_{0}}$.

Let $B_{1}$ and $\boldsymbol{B}_{2}$ represent the Banach space of all maps from $[v-1, v+b]_{\mathbb{N}_{v-1}}$ into $\mathbb{R}$ and $[\mu-1, \mu+b]_{\mathbb{N}_{\mu-1}}$ into $\mathbb{R}$, respectively. And $\|\cdot\|$ is the usual maximum norm. Define

$$
x:=B_{1} \times B_{2} .
$$


By equipping $\mathcal{X}$ with the norm

$$
\left\|\left(y_{1}, y_{2}\right)\right\|:=\left\|y_{1}\right\|+\left\|y_{2}\right\|
$$

it follows that $(\mathcal{X},\|\cdot\|)$ is a Banach space, see $[26,27]$.

Next, we wish to develop a representation for a solution of (1.4) as the fixed point of an appropriate operator on $\mathcal{X}$. To accomplish this, we present some straightforward adaptations of results from [24] that will be of use here. Since the proofs of these adaptations are evident, we do not include them.

Now, consider the operator $\mathcal{S}: \mathcal{X} \rightarrow \mathcal{X}$ defined by

$$
\mathcal{S}\left(y_{1}, y_{2}\right)\left(t_{1}, t_{2}\right):=\left(\mathcal{S}_{1}\left(y_{1}, y_{2}\right)\left(t_{1}\right), \quad \mathcal{S}_{2}\left(y_{1}, y_{2}\right)\left(t_{2}\right)\right)
$$

where we define $\mathcal{S}_{1}: \mathcal{X} \rightarrow \boldsymbol{B}_{1}$ by

$$
\mathcal{S}_{1}\left(y_{1}, y_{2}\right)\left(t_{1}\right):=\sum_{s=0}^{b+1} G_{1}\left(t_{1}, s\right) f\left(y_{1}(s+v-1), \quad y_{2}(s+\mu-1)\right)
$$

and $\mathcal{S}_{2}: X \rightarrow B_{2}$ by

$$
\mathcal{S}_{2}\left(y_{1}, y_{2}\right)\left(t_{2}\right):=\sum_{s=0}^{b+1} G_{2}\left(t_{2}, s\right) f\left(y_{1}(s+v-1), \quad y_{2}(s+\mu-1)\right)
$$

We claim that whenever $\left(y_{1}, y_{2}\right) \in \mathcal{X}$ is a fixed point of the operator $\mathcal{S}$, it follows that the pair of functions $y_{1}(t)$ and $y_{2}(t)$ are a solution to problem (1.4).

Lemma 2.8. Let $f, g: \mathbb{R} \times \mathbb{R} \rightarrow \mathbb{R}$. If $\left(y_{1}, y_{2}\right) \in \mathcal{X}$ is a fixed point of $\mathcal{S}$, then $\left(y_{1}, y_{2}\right)$ is a solution to problem (1.4).

Proof. Suppose that the operator $\mathcal{S}$ has a fixed point, say $\left(y_{1}, y_{2}\right) \in \mathcal{X}$. Let $\left(t_{1}, t_{2}\right) \in \mathbb{N}_{v-1} \times \mathbb{N}_{\mu-1}$, then we have

$$
y_{1}=\mathcal{S}_{1}\left(y_{1}, y_{2}\right)\left(t_{1}\right)
$$


where $\mathcal{S}_{1}$ is defined as in (2.15). It is easy to check that $\mathcal{S}_{1}\left(y_{1}, y_{2}\right)(v-2)=0$ and

$$
\begin{aligned}
& \Delta S_{1}\left(y_{1}, y_{2}\right)(v+b)=S_{1}\left(y_{1}, y_{2}\right)(v+b+1)-S_{1}\left(y_{1}, y_{2}\right)(v+b) \\
& =\sum_{s=0}^{b+1}\left(\frac{\Gamma(b+3)(v+b+1)^{\frac{v-1}{2}}}{\Gamma(v+b+1)}(v+s-1)^{\frac{v-2}{\underline{v}}}-(v+b-s)^{\frac{v-1}{}}\right) \\
& \times f\left(y_{1}(s+v-1), y_{2}(s+\mu-1)\right) \\
& -\sum_{s=0}^{b+1}\left(\frac{\Gamma(b+3)(v+b)^{\frac{v-1}{2}}}{\Gamma(v+b+1)}(v+b-s-1)^{\frac{v-2}{v}}-(v+b-s-1)^{\frac{v-1}{}}\right) \\
& \times f\left(y_{1}(s+v-1), y_{2}(s+\mu-1)\right) \\
& =\sum_{s=0}^{b+1}\left((v+b+1)(v+b-s-1)^{\frac{v-2}{u}}-(b+2)(v+b-s-1)^{\frac{v-2}{}}\right) \\
& \times f\left(y_{1}(s+v-1), y_{2}(s+\mu-1)\right) \\
& -\sum_{s=0}^{b+1}\left(\frac{(v+b-s) \Gamma(v+b-s)}{(b-s+1) \Gamma(b-s+1)}-\frac{\Gamma(v+b-s)}{\Gamma(b-s+1)}\right) \\
& \times f\left(y_{1}(s+v-1), y_{2}(s+\mu-1)\right) \\
& =\sum_{s=0}^{b+1}\left((v-1)(v+b-s-1)^{\frac{v-2}{2}}-\frac{(v-1) \Gamma(v+b-s)}{(b-s+1) \Gamma(b-s+1)}\right) \\
& \times f\left(y_{1}(s+v-1), y_{2}(s+\mu-1)\right) \\
& =\sum_{s=0}^{b+1}\left((v-1) \frac{\Gamma(v+b-s)}{\Gamma(b-s+2)}-\frac{(v-1) \Gamma(v+b-s)}{\Gamma(b-s+2)}\right) \\
& \times f\left(y_{1}(s+v-1), y_{2}(s+\mu-1)\right)=0 \text {, }
\end{aligned}
$$

so the boundary conditions are satisfied. Furthermore, we can check that $S_{1}\left(y_{1}, y_{2}\right)\left(t_{1}\right)$ satisfies the difference equation in (1.4). Since similar verifications may be made for $S_{2}\left(y_{1}, y_{2}\right)\left(t_{2}\right)$, the claim follows, which completes the proof.

\section{Main Results}

In this section, we will show that under certain conditions, problem (1.4) has at least one solution.

Let us now present the conditions that we will assume henceforth. We note that conditions (F1)-(F2) are similar to the conditions given by Henderson et al. [26]

$(\mathrm{F} 1): f, g \in C([0, \infty),[0, \infty))$

(F2) : $\lim _{\left(y_{1}, y_{2}\right) \rightarrow\left(0^{+}, 0^{+}\right)} \frac{f\left(y_{1}, y_{2}\right)}{y_{1}+y_{2}}=f^{*}, \lim _{\left(y_{1}, y_{2}\right) \rightarrow\left(0^{+}, 0^{+}\right)} \frac{g\left(y_{1}, y_{2}\right)}{y_{1}+y_{2}}=g^{*}$,

$$
\lim _{\left(y_{1}, y_{2}\right) \rightarrow(+\infty,+\infty)} \frac{f\left(y_{1}, y_{2}\right)}{y_{1}+y_{2}}=f^{* *}, \lim _{\left(y_{1}, y_{2}\right) \rightarrow(+\infty,+\infty)} \frac{g\left(y_{1}, y_{2}\right)}{y_{1}+y_{2}}=g^{* *},
$$


satisfying

$$
\begin{aligned}
\sum_{s=0}^{b+1} G_{1}(s+v-1, s)\left(f^{*}+\varepsilon\right) \leq \frac{1}{2}, & \sum_{s=0}^{b+1} G_{2}(s+\mu-1, s)\left(g^{*}+\varepsilon\right) \leq \frac{1}{2}, \\
\sum_{s=0}^{b+1} \gamma G_{1}(s+v-1, s)\left(f^{* *}-\varepsilon\right) \geq \frac{1}{2}, & \sum_{s=0}^{b+1} \gamma G_{2}(s+\mu-1, s)\left(g^{* *}-\varepsilon\right) \geq \frac{1}{2},
\end{aligned}
$$

for some $0<\varepsilon<\min \left\{f^{* *}, g^{* *}\right\}$.

Define the cone by

$$
\begin{aligned}
\mathcal{K}:=\{ & \left(y_{1}, y_{2}\right) \in \mathcal{X}: y_{1}, y_{2} \geq 0, \min _{\left(t_{1}, t_{2}\right) \in[(v-1) / 4,3(v+b) / 4] \times[(\mu-1) / 4,3(\mu+b) / 4]} \\
& \left.\times\left[y_{1}\left(t_{1}\right)+y_{2}\left(t_{2}\right)\right] \geq \gamma\left\|\left(y_{1}, y_{2}\right)\right\|\right\},
\end{aligned}
$$

where $\gamma=\min \left\{\gamma_{1}, \gamma_{2}\right\}$, for $i=1,2, \gamma_{i}$ is defined as in Lemma 2.7. Clearly, $\mathcal{K} \subseteq \mathcal{X}$. In order to show that $\mathcal{S}$ has a fixed point in $\mathcal{K}$, we must first demonstrate that $\mathcal{K}$ is invariant under $\mathcal{S}$, that is, $\mathcal{S}: \mathcal{K} \rightarrow \mathcal{K}$. We show this now.

Lemma 3.1. Assume that $f, g$ are nonnegative functions. Let $\mathcal{S}: \mathcal{X} \rightarrow \mathcal{X}$ be the operator defined as (2.14), then $\mathcal{S}: \mathcal{K} \rightarrow \mathcal{K}$.

Proof. Suppose that $\left(y_{1}, y_{2}\right) \in \mathcal{K}$. We claim that

$$
\min _{\left(t_{1}, t_{2}\right) \in[(v-1) / 4,3(v+b) / 4] \times[(\mu-1) / 4,3(\mu+b) / 4]}\left[\mathcal{S}_{1}\left(y_{1}, y_{2}\right)\left(t_{1}\right)+\mathcal{S}_{2}\left(y_{1}, y_{2}\right)\left(t_{2}\right)\right] \geq \gamma\left\|\mathcal{S}\left(y_{1}, y_{2}\right)\right\|
$$

In fact,

$$
\begin{aligned}
& \min _{t_{1} \in[(v-1) / 4,3(v+b) / 4]} \mathcal{S}_{1}\left(y_{1}, y_{2}\right)\left(t_{1}\right) \\
& \quad \geq \min _{t_{1} \in[(v-1) / 4,3(v+b) / 4]} \sum_{s=0}^{b+1} G_{1}\left(t_{1}, s\right) f\left(y_{1}(s+v-1), y_{2}(s+\mu-1)\right) \\
& \geq \gamma_{1} \sum_{s=0}^{b+1} G_{1}(s+v-1, s) f\left(y_{1}(s+v-1), y_{2}(s+\mu-1)\right) \\
& =\gamma_{1} \max _{t_{1} \in[v-1, v+b]_{\mathbb{N}_{v-1}}} \sum_{s=0}^{b+1} G_{1}\left(t_{1}, s\right) f\left(y_{1}(s+v-1), y_{2}(s+\mu-1)\right) \\
& =\gamma_{1}\left\|\mathcal{S}_{1}\left(y_{1}, y_{2}\right)\right\| .
\end{aligned}
$$

Similarly, we get

$$
\min _{t_{2} \in[(\mu-1) / 4,3(\mu+b) / 4]} \mathcal{S}_{2}\left(y_{1}, y_{2}\right)\left(t_{2}\right) \geq \gamma_{2}\left\|\mathcal{S}_{2}\left(y_{1}, y_{2}\right)\right\|
$$


Put $\gamma=\min \left\{\gamma_{1}, \gamma_{2}\right\}$. Consequently, from (3.5) and (3.6), we obtain

$$
\begin{aligned}
& \min _{\left(t_{1}, t_{2}\right) \in[(v-1) / 4,3(v+b) / 4] \times[(\mu-1) / 4,3(\mu+b) / 4]}\left[\mathcal{S}_{1}\left(y_{1}, y_{2}\right)\left(t_{1}\right)+\mathcal{S}_{2}\left(y_{1}, y_{2}\right)\left(t_{2}\right)\right] \\
& \geq_{t_{1} \in[(v-1) / 4,3(v+b) / 4]} \mathcal{S}_{1}\left(y_{1}, y_{2}\right)\left(t_{1}\right) \\
& +\min _{t_{2} \in[(\mu-1) / 4,3(\mu+b) / 4]} \mathcal{S}_{2}\left(y_{1}, y_{2}\right)\left(t_{2}\right) \\
& \geq \gamma_{1}\left\|S_{1}\left(y_{1}, y_{2}\right)\right\|+\gamma_{2}\left\|S_{2}\left(y_{1}, y_{2}\right)\right\| \\
& \geq r\left(\left\|\mathcal{S}_{1}\left(y_{1}, y_{2}\right)\right\|+\left\|S_{2}\left(y_{1}, y_{2}\right)\right\|\right) \\
& =r\left\|\mathcal{S}\left(y_{1}, y_{2}\right)\right\| \text {. }
\end{aligned}
$$

So, for $\left(y_{1}, y_{2}\right) \in \mathcal{K}$, we find

$$
\min _{\left(t_{1}, t_{2}\right) \in[(v-1) / 4,3(v+b) / 4] \times[(\mu-1) / 4,3(\mu+b) / 4]}\left[\mathcal{S}_{1}\left(y_{1}, y_{2}\right)\left(t_{1}\right)+\mathcal{S}_{2}\left(y_{1}, y_{2}\right)\left(t_{2}\right)\right] \geq \gamma\left\|\mathcal{S}\left(y_{1}, y_{2}\right)\right\|
$$

Therefore, from (3.8), whenever we get $\left(y_{1}, y_{2}\right) \in \mathcal{K}$, it follows that $\mathcal{S}\left(y_{1}, y_{2}\right) \in \mathcal{K}$. This completes the proof.

We next recall the Guo-Krasnosel'kii fixed-point theorem and Schauder fixed-point theorem, which we will use to prove the main results.

Lemma 3.2. ([21]) Let $\boldsymbol{B}$ be a Banach space, and let $\mathcal{K} \subseteq B$ be a cone. Assume that $\Omega_{1}$ and $\Omega_{2}$ are open subsets contained in $乃$ such that $0 \in \Omega_{1}$ and $\overline{\Omega_{1}} \subseteq \Omega_{2}$. Assume, further, that $T: \mathcal{K} \cap\left(\overline{\Omega_{2}} \backslash \Omega_{1}\right) \rightarrow \mathcal{K}$ is a completely continuous operator. If either

(1) $\|T y\| \leq\|y\|$ for $y \in \mathcal{K} \cap \partial \Omega_{1}$ and $\|T y\| \geq\|y\|$ for $y \in \mathcal{K} \cap \partial \Omega_{2}$,

(2) or $\|T y\| \geq\|y\|$ for $y \in \mathcal{K} \cap \partial \Omega_{1}$ and $\|T y\| \leq\|y\|$ for $y \in \mathcal{K} \cap \partial \Omega_{2}$,

then $T$ has at least one fixed point in $\mathcal{K} \cap\left(\overline{\Omega_{2}} \backslash \Omega_{1}\right)$.

Lemma 3.3. ([22]) (Schauder fixed-point theorem) Suppose that $\mathcal{X}$ is a Banach space. Let $\mathcal{K}$ be a bounded closed-convex set of $\mathcal{X}$, and let $T: \mathcal{K} \rightarrow \mathcal{K}$ be a completely continuous operator, then $T$ has at least one fixed point in $\mathcal{K}$.

Theorem 3.4. Suppose that conditions (F1)-(F2) hold, then problem (1.4) has at least one solution.

Proof. From Lemma 3.1, we know that $\mathcal{S}: \mathcal{K} \rightarrow \mathcal{K}$. Note that $\mathcal{S}$ is a summation operator on a discrete finite set. Hence, $\mathcal{S}$ is a completely continuous operator. that

By conditions (F1) and (F2), for $\varepsilon$ given in (F2), there exists some constant $r_{1}>0$, such

$$
\begin{aligned}
& f\left(y_{1}, y_{2}\right) \leq\left(f^{*}+\varepsilon\right)\left(y_{1}+y_{2}\right), \\
& g\left(y_{1}, y_{2}\right) \leq\left(g^{*}+\varepsilon\right)\left(y_{1}+y_{2}\right),
\end{aligned}
$$

whenever $\left\|\left(y_{1}, y_{2}\right)\right\|<r_{1}$. 
Setting

$$
\Omega_{1}:=\left\{\left(y_{1}, y_{2}\right) \in \mathcal{X}:\left\|\left(y_{1}, y_{2}\right)\right\|<r_{1}\right\}
$$

Then, for $\left(y_{1}, y_{2}\right) \in \mathcal{K} \cap \partial \Omega_{1}$, by Lemma 2.7, (F2) and (3.9), we find that

$$
\begin{aligned}
\left\|\mathcal{S}_{1}\left(y_{1}, y_{2}\right)\right\| & =\max _{t_{1} \in[v-1, v+b]_{N_{v-1}}} \sum_{s=0}^{b+1} G_{1}\left(t_{1}, s\right) f\left(y_{1}(s+v-1), y_{2}(s+\mu-1)\right) \\
& \leq \sum_{s=0}^{b+1} G_{1}(s+v-1, s)\left(f^{*}+\varepsilon\right)\left(y_{1}+y_{2}\right) \\
& \leq\left\|\left(y_{1}, y_{2}\right)\right\| \sum_{s=0}^{b+1} G_{1}(s+v-1, s)\left(f^{*}+\varepsilon\right) \\
& \leq \frac{1}{2} r_{1}=\frac{1}{2}\left\|\left(y_{1}, y_{2}\right)\right\| .
\end{aligned}
$$

Similar, by (3.10), it can be shown that

$$
\left\|\mathcal{S}_{2}\left(y_{1}, y_{2}\right)\right\| \leq \frac{1}{2}\left\|\left(y_{1}, y_{2}\right)\right\|
$$

Thus, putting (3.12) and (3.13) together, for $\left(y_{1}, y_{2}\right) \in \mathcal{K} \cap \partial \Omega_{1}$, we have

$$
\left\|\mathcal{S}\left(y_{1}, y_{2}\right)\right\| \leq \frac{1}{2}\left\|\left(y_{1}, y_{2}\right)\right\|+\frac{1}{2}\left\|\left(y_{1}, y_{2}\right)\right\|=\left\|\left(y_{1}, y_{2}\right)\right\|
$$

Now, for the above $\varepsilon$, by (F2), we can find a constant $r^{* *}>0$ such that

$$
\begin{aligned}
& f\left(y_{1}, y_{2}\right) \geq\left(f^{* *}-\varepsilon\right)\left(y_{1}+y_{2}\right), \\
& g\left(y_{1}, y_{2}\right) \geq\left(g^{* *}-\varepsilon\right)\left(y_{1}+y_{2}\right),
\end{aligned}
$$

whenever $\left\|y_{1}+y_{2}\right\| \geq r^{* *}$. Let

$$
\frac{1}{2} r^{* *} \leq r_{2} \leq \min \left\{\sum_{s=0}^{b+1} G_{1}(s+v-1, s)\left(f^{* *}-\varepsilon\right) \gamma r^{* *}, \sum_{s=0}^{b+1} G_{2}(s+\mu-1, s)\left(g^{* *}-\varepsilon\right) \gamma r^{* *}\right\} .
$$

Moreover, put

$$
\Omega_{2}:=\left\{\left(y_{1}, y_{2}\right) \in \mathcal{X}:\left\|\left(y_{1}, y_{2}\right)\right\|<2 r_{2}\right\}
$$

If $\left(y_{1}, y_{2}\right) \in \mathcal{K} \cap \partial \Omega_{2}$, then

$$
y_{1}\left(t_{1}\right)+y_{2}\left(t_{2}\right) \geq \min _{\left(t_{1}, t_{2}\right) \in[(v-1) / 4,3(v+b) / 4] \times[(\mu-1) / 4,3(\mu+b) / 4]}\left[y_{1}\left(t_{1}\right)+y_{2}\left(t_{2}\right)\right] \geq \gamma\left\|\left(y_{1}, y_{2}\right)\right\|
$$


Thus, from (3.15) and (3.19), we obtain

$$
\begin{aligned}
\left\|\mathcal{S}_{1}\left(y_{1}, y_{2}\right)\right\| & =\max _{t_{1} \in[v-1, v+b]_{\mathbb{N}_{v-1}}} \sum_{s=0}^{b+1} G_{1}\left(t_{1}, s\right) f\left(y_{1}(s+v-1), y_{2}(s+\mu-1)\right) \\
& \geq \sum_{s=0}^{b+1} G_{1}(s+v-1, s)\left(f^{* *}-\varepsilon\right)\left(y_{1}+y_{2}\right) \\
& \geq \sum_{s=0}^{b+1} G_{1}(s+v-1, s)\left(f^{* *}-\varepsilon\right) \gamma\left\|\left(y_{1}, y_{2}\right)\right\| \\
& \geq r_{2}=\frac{1}{2}\left\|\left(y_{1}, y_{2}\right)\right\|
\end{aligned}
$$

Similarly, by (3.16) and (3.19), we have

$$
\left\|\mathcal{S}_{2}\left(y_{1}, y_{2}\right)\right\| \geq \frac{1}{2}\left\|\left(y_{1}, y_{2}\right)\right\|
$$

So, from (3.20) and (3.21), we get

$$
\left\|\mathcal{S}\left(y_{1}, y_{2}\right)\right\| \geq\left\|\left(y_{1}, y_{2}\right)\right\|
$$

whenever $\left(y_{1}, y_{2}\right) \in \mathcal{K} \cap \partial \Omega_{2}$.

Thus, by (3.14) and (3.22), we get that all of the hypotheses of Lemma 3.2 are satisfied. Consequently, we conclude that $\mathcal{S}$ has a fixed point, say $\left(y_{1}^{*}, y_{2}^{*}\right) \in \mathcal{K}$. Then the theorem is proved.

Theorem 3.5. Let $f, g: \mathbb{R} \times \mathbb{R} \rightarrow \mathbb{R}$ be continuous functions. Suppose that the following conditions are satisfied:

(i) there exist nonnegative functions $a, h, p, q \in C\left(\mathbb{R}, \mathbb{R}^{+}\right)$such that

$$
\left|f\left(y_{1}, y_{2}\right)\right| \leq a\left(y_{1}\right)+p\left(y_{2}\right),\left|g\left(y_{1}, y_{2}\right)\right| \leq h\left(y_{1}\right)+q\left(y_{2}\right)
$$

(ii) Consider the following:

$$
\lim _{|y| \rightarrow+\infty} \frac{p(y)}{|y|}<A, \quad \lim _{|y| \rightarrow+\infty} \frac{q(y)}{|y|}<B
$$

where $A=\left(2 \max _{t \in[v-1, v+b+1]} \sum_{s=0}^{b+1} G_{1}(t, s)\right)^{-1}, B=\left(2 \max _{t \in[\mu-1, \mu+b+1]} \sum_{s=0}^{b+1} G_{2}(t, s)\right)^{-1}$, then boundary value problem (1.4) has at least one solution $\left(y_{1}^{* *}, y_{2}^{* *}\right)$.

Proof. Let $\varepsilon_{1}=(1 / 2)\left(A-\lim _{|y| \rightarrow+\infty} p(y) /|y|\right)$. By (ii), there exists a $c_{1}>0$, such that $p\left(y_{2}\right)<$ $\left(A-\varepsilon_{1}\right)\left|y_{2}\right|$, for $\left|y_{2}\right| \geq c_{1}$. Set $M=\max \left\{p\left(y_{2}\right):\left|y_{2}\right| \leq c_{1}\right\}$, then there exists a $c_{2}>c_{1}$, such that $M / c_{2} \leq A-\varepsilon_{1}$, so we have $p\left(y_{2}\right)<\left(A-\varepsilon_{1}\right) c_{2}$, for $\left|y_{2}\right| \leq c_{2}$. 
Let $\varepsilon_{2}=(1 / 2)\left(B-\lim _{|y| \rightarrow+\infty} q(y) /|y|\right)$. In the same way, there exists a constant $c_{3}>0$, such that $q\left(y_{2}\right)<\left(B-\varepsilon_{2}\right) c_{3},\left|y_{2}\right| \leq c_{3}$. Define

$$
\begin{gathered}
e_{1}=\max _{t_{1} \in[v-1, v+b+1]} \sum_{s=0}^{b+1} G_{1}\left(t_{1}, s\right) a\left(y_{1}\right), \quad e_{2}=\sum_{t_{2} \in[\mu-1, \mu+b+1]} \sum_{s=0}^{b+1} G_{2}\left(t_{2}, s\right) h\left(y_{1}\right), \\
c=\max \left\{c_{2}, c_{3}, \frac{2 A}{\varepsilon_{1}} e_{1}, \frac{2 B}{\varepsilon_{2}} e_{2}\right\}, \\
U=\left\{\begin{array}{l}
\left(y_{1}, y_{2}\right) \mid\left(y_{1}, y_{2}\right) \in X,\left\|\left(y_{1}, y_{2}\right)\right\| \leq c,\left(t_{1}, t_{2}\right) \in[v-1, v+b+1]_{\mathbb{N}_{v-1}} \\
\left.\times[\mu-1, \mu+b+1]_{\mathbb{N}_{\mu-1}}\right\} .
\end{array}\right.
\end{gathered}
$$

Then $U$ is a bounded closed-convex set of $\mathcal{X} \times \mathcal{X}$, and for any $\left(y_{1}, y_{2}\right) \in U$, we have $p\left(y_{2}\right)<$ $\left(A-\varepsilon_{1}\right) c, q\left(y_{2}\right)<\left(B-\varepsilon_{2}\right) c$. Next, we prove $\mathcal{S}: U \rightarrow U$, where $\mathcal{S}$ is defined as (2.14).

For $\left(y_{1}, y_{2}\right) \in U$, from (i) and (sii), we obtain

$$
\begin{aligned}
\mathcal{S}_{1}\left(y_{1}, y_{2}\right)\left(t_{1}\right) & =\sum_{s=0}^{b+1} G_{1}\left(t_{1}, s\right) f\left(y_{1}, y_{2}\right) \\
& \leq \sum_{s=0}^{b+1} G_{1}\left(t_{1}, s\right) a\left(y_{1}\right)+\sum_{s=0}^{b+1} G_{1}\left(t_{1}, s\right) p\left(y_{2}\right) \\
& \leq e_{1}+\left(A-\varepsilon_{1}\right) c \sum_{s=0}^{b+1} G_{1}\left(t_{1}, s\right) \\
& \leq \frac{\varepsilon_{1}}{2 A} c+\frac{1}{2}\left(1-\frac{\varepsilon_{1}}{A}\right) c=\frac{1}{2} c,
\end{aligned}
$$

similarly,

$$
S_{2}\left(y_{1}, y_{2}\right)\left(t_{2}\right) \leq \frac{\varepsilon_{2}}{2 B} c+\frac{1}{2}\left(1-\frac{\varepsilon_{2}}{B}\right) c=\frac{1}{2} c
$$

Thus, from (3.26) and (3.27), we have $\left\|\mathcal{S}\left(y_{1}, y_{2}\right)\right\| \leq c$. Then, $\mathcal{S}: U \rightarrow U$.

Note that $\mathcal{S}$ is a summation operator on a discrete finite set. Hence, $\mathcal{S}$ is trivially completely continuous. By Schauder fixed-point theorem, the boundary problem value problem (1.4) has at least one solution, say $\left(y_{1}^{* *}, y_{2}^{* *}\right)$. This completes the proof.

\section{Examples}

In this section, we will present some examples to illustrate the main results. 
Example 4.1. Suppose that $v=3 / 2, \mu=5 / 4, \eta_{1}=1 / 1000, \eta_{2}=10 / \gamma, \gamma=\min \left\{\gamma_{1}, \gamma_{2}\right\}$, and $\gamma_{1}$, $\gamma_{2}$ are defined as in Lemma $2.7, b=2$. Let $f\left(y_{1}, y_{2}\right):=\left(\left(1 / 1000 e^{-y_{2}}\right)+\left(1000 / \gamma e^{1 / y_{1}}\right)\right)\left(y_{1}+y_{2}\right)$ and $g(y):=\left(\left(1 / 2000 e^{-y_{1}}\right)+\left(1000 / \gamma e^{1 / y_{2}}\right)\right)\left(y_{1}+y_{2}\right)$, then (1.4) becomes

$$
\begin{gathered}
-\Delta^{3 / 2} y_{1}(t)=f\left(y_{1}\left(t+\frac{1}{2}\right), y_{2}\left(t+\frac{1}{4}\right)\right), \text { for } t \in[0,3]_{\mathbb{N}_{0}}, \\
-\Delta^{5 / 4} y_{2}(t)=g\left(y_{1}\left(t+\frac{1}{2}\right), y_{2}\left(t+\frac{1}{4}\right)\right), \text { for } t \in[0,3]_{\mathbb{N}_{0}} \\
y_{1}\left(-\frac{1}{2}\right)=\Delta y_{1}\left(\frac{7}{2}\right)=0, \\
y_{2}\left(-\frac{3}{4}\right)=\Delta y_{2}\left(\frac{13}{4}\right)=0 .
\end{gathered}
$$

In this case,

$$
\begin{aligned}
\lim _{\left(y_{1}, y_{2}\right) \rightarrow\left(0^{+}, 0^{+}\right)} \frac{f\left(y_{1}, y_{2}\right)}{y_{1}+y_{2}} & =\frac{1}{1000}=f^{*}, \quad \lim _{\left(y_{1}, y_{2}\right) \rightarrow\left(+\infty, y_{2}\right) \rightarrow\left(0^{+}, 0^{+}\right)} \frac{g\left(y_{1}, y_{2}\right)}{y_{1}+y_{2}}=\frac{1}{2000}=g^{*}, \\
\lim _{\left(y_{1}, y_{2}\right) \rightarrow(+\infty,+\infty)} \frac{f\left(y_{1}, y_{2}\right)}{y_{1}+y_{2}}= & \frac{1000}{r}=f^{* *}, \quad \lim _{y_{1}+y_{2}}=\frac{1000}{r}=g^{* *}, \\
\sum_{s=0}^{b+1} G_{1}(s+v-1, s) & =\sum_{s=0}^{b+1} \frac{\Gamma(b+3)(s+v-1) \frac{v-1}{\Gamma(v+b+1)}(v+b-s-1)^{\frac{v-2}{2}}}{} \\
& =\sum_{s=0}^{b+1} \frac{\Gamma(b+3) \Gamma(s+v) \Gamma(v+b-s)}{\Gamma(v+b+1) \Gamma(s+1) \Gamma(b-s+2)} \\
& =\sum_{s=0}^{b+1} \frac{(b+2)(b+1) \cdots(b-s+2)(s+v-1) \cdots(s+1)}{(v+b)(v+b-1) \cdots(v+b-s)} \\
& \leq \sum_{s=0}^{b+1}(s+v-1) \cdots(s+1) \\
& \leq(b+2)(b+v)(b+2)=4 \times \frac{7}{2} \times 4=56 .
\end{aligned}
$$

Similarly, we get

$$
\sum_{s=0}^{b+1} G_{2}(s+\mu-1, s) \leq(b+2)(b+\mu)(b+2)=52 .
$$


Thus,

$$
\begin{aligned}
& \sum_{s=0}^{b+1} G_{1}(s+v-1, s)\left(f^{*}+\eta_{1}\right) \leq 56 \times\left(\frac{1}{1000}+\frac{1}{1000}\right) \leq \frac{1}{2} \\
& \sum_{s=0}^{b+1} G_{2}(s+v-1, s)\left(g^{*}+\eta_{1}\right) \leq 52 \times\left(\frac{1}{2000}+\frac{1}{1000}\right) \leq \frac{1}{2} .
\end{aligned}
$$

On the other hand,

$$
\begin{aligned}
\sum_{s=0}^{b+1} G_{1}(s+v-1, s) & =\sum_{s=0}^{b+1} \frac{(b+2)(b+1) \cdots(b-s+2)(s+v-1) \cdots(s+1)}{(v+b)(v+b-1) \cdots(v+b-s)} \\
& \geq \sum_{s=0}^{b+1}(b+2)(b+1) \cdots(b-s+2)(s+v-1) \cdots(s+1) \\
& \geq(b+2)(b+2)(v-1)=2 \times 2 \times \frac{1}{2}=2,
\end{aligned}
$$

similarly,

$$
\sum_{s=0}^{b+1} G_{2}(s+\mu-1, s) \geq(b+2)(b+2)(\mu-1) \geq 4 \times 4 \times \frac{1}{4}=1 .
$$

Then,

$$
\begin{gathered}
\sum_{s=0}^{b+1} r G_{1}(s+v-1, s)\left(f^{* *}-\eta_{2}\right) \geq 2 \gamma \times\left(\frac{1000}{r}-\frac{10}{r}\right)=1980 \geq \frac{1}{2} \\
\sum_{s=0}^{b+1} r G_{2}(s+\mu-1, s)\left(g^{* *}-\eta_{2}\right) \geq \gamma \times\left(\frac{1000}{r}-\frac{10}{r}\right)=990 \geq \frac{1}{2} .
\end{gathered}
$$

Thus, the conditions of Theorem 3.4 are satisfied. So, we obtain that the problem (4.1) has at least one solution.

Example 4.2. Suppose that $v=5 / 3, \mu=7 / 4$, and $b=3$. Let $f\left(y_{1}, y_{2}\right):=y_{1}+(A / 4) y_{2} e^{-\left|y_{2}\right|}$, $g\left(y_{1}, y_{2}\right):=y_{1}+(B / 5) y_{2} e^{-\left|y_{2}\right|}, a\left(y_{1}\right)=\left|y_{1}\right|, h\left(y_{1}\right)=2\left|y_{1}\right|, p\left(y_{2}\right)=(A / 3)\left|y_{2}\right|\left(e^{-\left|y_{2}\right|}+1\right)$, and $q\left(y_{2}\right)=(B / 4)\left|y_{2}\right|\left(e^{-\left|y_{2}\right|}+1\right), y_{1}, y_{2} \in \mathbb{R}^{+}$, where

$$
A=\left(2 \max _{t \in[v-1, v+b+1]} \sum_{s=0}^{b+1} G_{1}(t, s)\right)^{-1}, \quad B=\left(2 \max _{t \in[\mu-1, \mu+b+1]} \sum_{s=0}^{b+1} G_{2}(t, s)\right)^{-1}
$$


Then problem (1.4) is

$$
\begin{aligned}
-\Delta^{5 / 3} y_{1}(t) & =y_{1}\left(t+\frac{2}{3}\right)+\frac{A}{4} y_{2}\left(t+\frac{3}{4}\right) e^{-\left|y_{2}(t+(3 / 4))\right|}, \quad \text { for } t \in[0,4]_{\mathbb{N}_{0}} \\
-\Delta^{7 / 4} y_{1}(t) & =y_{1}\left(t+\frac{2}{3}\right)+\frac{B}{5} y_{2}\left(t+\frac{3}{4}\right) e^{-\left|y_{2}(t+(3 / 4))\right|}, \quad \text { for } t \in[0,4]_{\mathbb{N}_{0}} \\
y_{1}\left(-\frac{1}{3}\right) & =\Delta y_{1}\left(\frac{14}{3}\right)=0 \\
y_{2}\left(-\frac{1}{4}\right) & =\Delta y_{2}\left(\frac{19}{4}\right)=0 .
\end{aligned}
$$

It is clear that $f, g, a, h, p$, and $q$ satisfy the conditions of Theorem 3.5. Thus, by Theorem 3.5, we deduce that problem (4.10) has at least one solution.

\section{Acknowledgments}

The authors sincerely thank the reviewers for their valuable suggestions and useful comments that have lead to the present improved version of the original paper. This research is supported by the Natural Science Foundation of China $(11071143,60904024,61174217)$, Natural Science Outstanding Youth Foundation of Shandong Province (JQ201119), Shandong Provincial Natural Science Foundation (ZR2010AL002, ZR2009AL003), and the Natural Science Foundation of Educational Department of Shandong Province (J11LA01).

\section{References}

[1] Z. Bai and H. Lü, "Positive solutions for boundary value problem of nonlinear fractional differential equation," Journal of Mathematical Analysis and Applications, vol. 311, no. 2, pp. 495-505, 2005.

[2] M. Benchohra, S. Hamani, and S. K. Ntouyas, "Boundary value problems for differential equations with fractional order and nonlocal conditions," Nonlinear Analysis, vol. 71, no. 7-8, pp. 2391-2396, 2009.

[3] K. Diethelm and N. J. Ford, "Analysis of fractional differential equations," Journal of Mathematical Analysis and Applications, vol. 265, no. 2, pp. 229-248, 2002.

[4] X. Xu, D. Jiang, and C. Yuan, "Multiple positive solutions for the boundary value problem of a nonlinear fractional differential equation," Nonlinear Analysis, vol. 71, no. 10, pp. 4676-4688, 2009.

[5] Y. Zhao, S. Sun, Z. Han, and Q. Li, "Positive solutions to boundary value problems of nonlinear fractional differential equations," Abstract and Applied Analysis, vol. 2011, Article ID 390543, 16 pages, 2011.

[6] Y. Zhao, S. Sun, Z. Han, and Q. Li, "The existence of multiple positive solutions for boundary value problems of nonlinear fractional differential equations," Communications in Nonlinear Science and Numerical Simulation, vol. 16, no. 4, pp. 2086-2097, 2011.

[7] Y. Zhao, S. Sun, Z. Han, and M. Zhang, "Positive solutions for boundary value problems of nonlinear fractional differential equations," Applied Mathematics and Computation, vol. 217, no. 16, pp. 6950-6958, 2011.

[8] Y. Zhao, S. Sun, Z. Han, and Q. Li, "Theory of fractional hybrid differential equations," Computers $\mathcal{E}$ Mathematics with Applications, vol. 62, no. 3, pp. 1312-1324, 2011.

[9] Y. Zhao, S. Sun, Z. Han, and W. Feng, "Positive solutions for a coupled system of nonlinear differential equations of mixed fractional orders," Advances in Difference Equations, vol. 2011, article 10, 13 pages, 2011.

[10] Y. Zhou, F. Jiao, and J. Li, "Existence and uniqueness for $p$-type fractional neutral differential equations," Nonlinear Analysis, vol. 71, no. 7-8, pp. 2724-2733, 2009. 
[11] Y. Zhou, F. Jiao, and J. Li, “Existence and uniqueness for fractional neutral differential equations with infinite delay," Nonlinear Analysis, vol. 71, no. 7-8, pp. 3249-3256, 2009.

[12] Y. Zhou and F. Jiao, "Nonlocal Cauchy problem for fractional evolution equations," Nonlinear Analysis, vol. 11, no. 5, pp. 4465-4475, 2010.

[13] C. S. Goodrich, "Existence of a positive solution to a class of fractional differential equations," Applied Mathematics Letters, vol. 23, no. 9, pp. 1050-1055, 2010.

[14] C. S. Goodrich, "Existence of a positive solution to systems of differential equations of fractional order," Computers \& Mathematics with Applications, vol. 62, no. 3, pp. 1251-1268, 2011.

[15] F. M. Atici and P. W. Eloe, "A transform method in discrete fractional calculus," International Journal of Difference Equations, vol. 2, no. 2, pp. 165-176, 2007.

[16] F. M. Atici and P. W. Eloe, "Fractional q-calculus on a time scale," Journal of Nonlinear Mathematical Physics, vol. 14, no. 3, pp. 333-344, 2007.

[17] F. M. Atıc1 and P. W. Eloe, "Two-point boundary value problems for finite fractional difference equations," Journal of Difference Equations and Applications, vol. 17, no. 4, pp. 445-456, 2011.

[18] F. M. Atici and P. W. Eloe, "Initial value problems in discrete fractional calculus," Proceedings of the American Mathematical Society, vol. 137, no. 3, pp. 981-989, 2009.

[19] C. S. Goodrich, "Continuity of solutions to discrete fractional initial value problems," Computers $\mathcal{E}$ Mathematics with Applications, vol. 59, no. 11, pp. 3489-3499, 2010.

[20] C. S. Goodrich, "Existence and uniqueness of solutions to a fractional difference equation with nonlocal conditions," Computers \& Mathematics with Applications, vol. 61, no. 2, pp. 191-202, 2011.

[21] R. P. Agarwal, M. Meehan, and D. O’Regan, Fixed Point Theory and Applications, Cambridge University Press, Cambridge, UK, 2001.

[22] J. Schauder, "Der Fixpunktsatz in Funktionalräumen," Studia Mathematica, vol. 2, pp. 171-180, 1930.

[23] Y. Pan and Z. Han, "Existence of solutions for a coupled system of boundary value probelm of nonlinear fractional differential equations," in Proceedings of the 5th International Congress on Mathematical Biology, vol. 1, pp. 109-114, 2011.

[24] C. S. Goodrich, "Solutions to a discrete right-focal fractional boundary value problem," International Journal of Difference Equations, vol. 5, no. 2, pp. 195-216, 2010.

[25] C. S. Goodrich, "Existence of a positive solution to a system of discrete fractional boundary value problems," Applied Mathematics and Computation, vol. 217, no. 9, pp. 4740-4753, 2011.

[26] J. Henderson, S. K. Ntouyas, and I. K. Purnaras, "Positive solutions for systems of nonlinear discrete boundary value problems," Journal of Difference Equations and Applications, vol. 15, no. 10, pp. 895-912, 2009.

[27] D. R. Dunninger and H. Wang, "Existence and multiplicity of positive solutions for elliptic systems," Nonlinear Analysis, vol. 29, no. 9, pp. 1051-1060, 1997.

[28] C. S. Goodrich, "On positive solutions to nonlocal fractional and integer-order difference equations," Applicable Analysis and Discrete Mathematics, vol. 5, no. 1, pp. 122-132, 2011.

[29] C. S. Goodrich, "On discrete sequential fractional boundary value problems," Journal of Mathematical Analysis and Applications, vol. 385, no. 1, pp. 111-124, 2012.

[30] R. A. C. Ferreira, "Positive solutions for a class of boundary value problems with fractional $q$ differences," Computers \& Mathematics with Applications, vol. 61, no. 2, pp. 367-373, 2011.

[31] N. R. O. Bastos, R. A. C. Ferreira, and D. F. M. Torres, "Discrete-time fractional variational problems," Signal Processing, vol. 91, no. 3, pp. 513-524, 2011.

[32] F. M. Atıcı and S. Şengül, "Modeling with fractional difference equations," Journal of Mathematical Analysis and Applications, vol. 369, no. 1, pp. 1-9, 2010. 


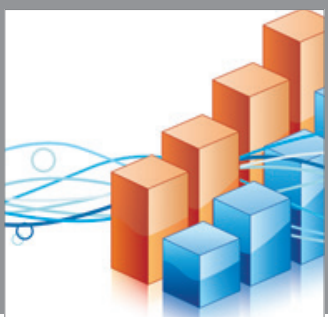

Advances in

Operations Research

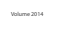

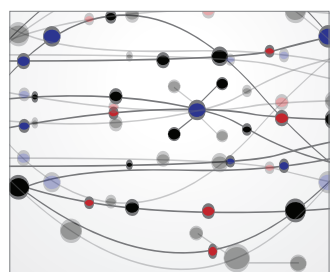

\section{The Scientific} World Journal
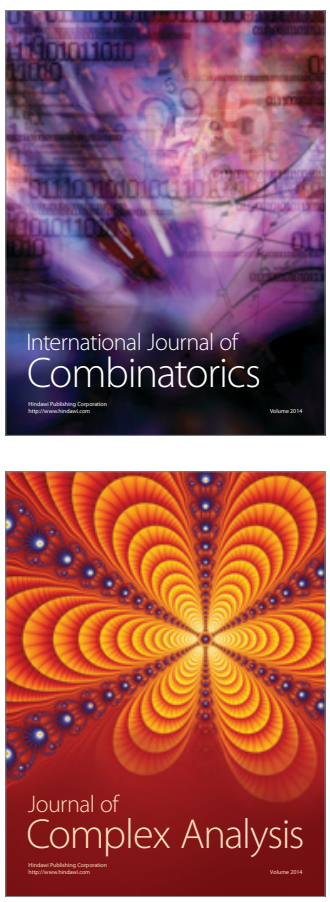

International Journal of

Mathematics and

Mathematical

Sciences
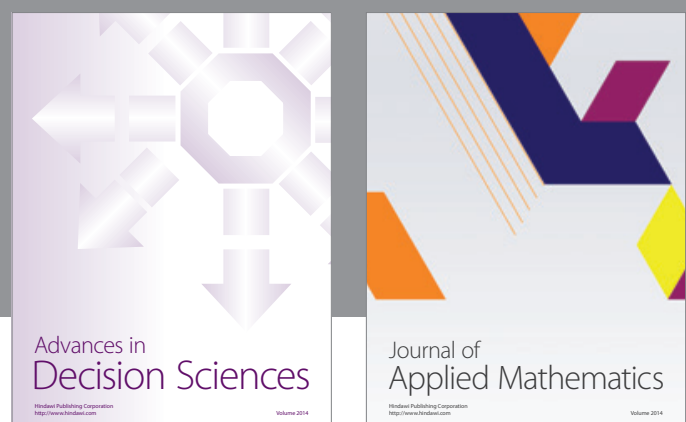

Journal of

Applied Mathematics
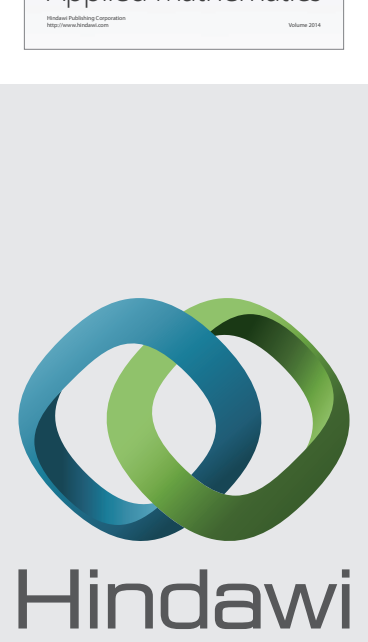

Submit your manuscripts at http://www.hindawi.com
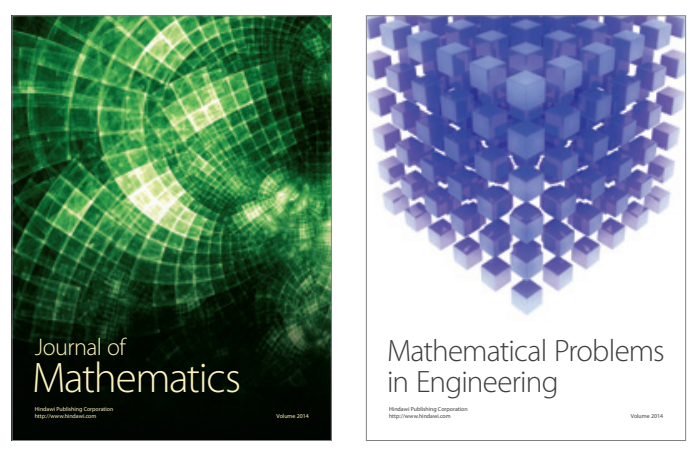

Mathematical Problems in Engineering
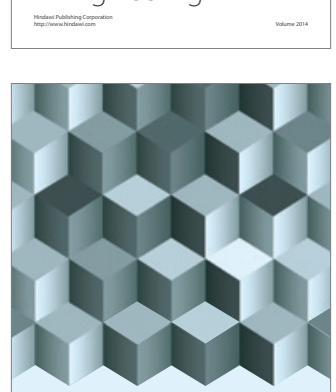

Journal of

Function Spaces
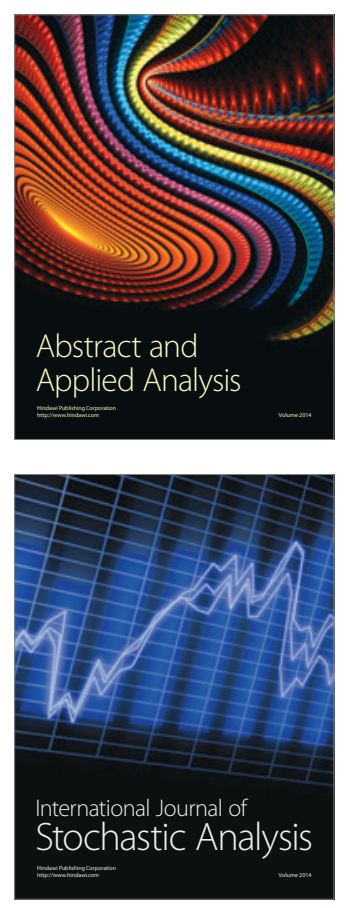

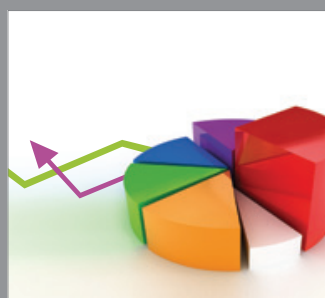

ournal of

Probability and Statistics

Promensencen
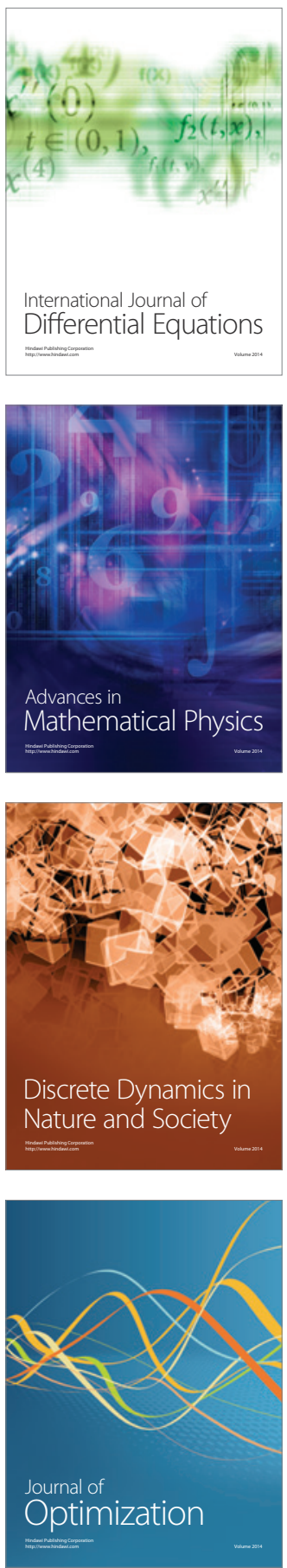